\title{
BMJ Open Do sociodemographic variables moderate effects of an internet intervention for mild to moderate depressive symptoms? An exploratory analysis of a randomised controlled trial (EVIDENT) including 1013 participants
}

\author{
Sandra Nolte (D) , ${ }^{1}$ Ljoudmila Busija, ${ }^{2}$ Thomas Berger (D) , ${ }^{3}$ Björn Meyer, ${ }^{4,5}$ \\ Steffen Moritz, ${ }^{6}$ Matthias Rose, ${ }^{1}$ Johanna Schröder, ${ }^{6}$ Christina Späth-Nellissen, ${ }^{7}$ \\ Jan Philipp Klein (i) $^{7}$
}

To cite: Nolte S, Busija L, Berger T, et al. Do sociodemographic variables moderate effects of an internet intervention for mild to moderate depressive symptoms? An exploratory analysis of a randomised controlled trial (EVIDENT) including 1013 participants. BMJ Open 2021;11:e041389. doi:10.1136/ bmjopen-2020-041389

- Prepublication history for this paper is available online. To view these files, please visit the journal online (http://dx.doi org/10.1136/bmjopen-2020041389).

Received 06 June 2020 Revised 26 November 2020 Accepted 28 December 2020

Check for updates

(C) Author(s) (or their employer(s)) 2021. Re-use permitted under CC BY-NC. No commercial re-use. See rights and permissions. Published by BMJ.

For numbered affiliations see end of article.

Correspondence to

Dr Sandra Nolte;

sandra.nolte@charite.de

\section{ABSTRACT}

Objective To explore the moderating effects of sociodemographic variables on treatment benefits received from participating in an internet intervention for depression.

Design Randomised, assessor-blind, controlled trial.

Setting Online intervention, with participant recruitment using multiple settings, including inpatient and outpatient medical and psychological clinics, depression online forums, health insurance companies and the media (eg, newspaper, radio).

Participants The EVIDENT trial included 1013 participants with mild to moderate depressive symptoms.

Interventions The intervention group subjects $(\mathrm{n}=509)$ received an online intervention (Deprexis) in addition to care as usual (CAU), while 504 participants received CAU alone.

Methods To explore subgroup differences, moderating effects were investigated using linear regression models based on intention-to-treat analyses. Moderating effects included sex, age, educational attainment, employment status, relationship status and lifetime frequency of episodes.

Primary and secondary outcome measures The primary endpoint was change in self-rated depression severity measured by the Patient Health Questionnaire-9 (PHQ-9), comparing baseline versus 12-week post-test assessment. Secondary outcome measures were the Hamilton Rating Scale for Depression and the Quick Inventory of Depressive Symptoms each at 12 weeks and at 6 and 12 months, and PHQ-9 at 6 and 12 months, respectively. In this article, we focus on the primary outcome measure only.

Results Between-group differences were observed in post-test scores, indicating the effectiveness of Deprexis. While the effects of the intervention could be demonstrated across all subgroups, some showed larger between-group differences than others. However, after exploring the moderating effects based on linear regression models, none of the selected variables was found to be moderating treatment outcomes.
Strengths and limitations of this study

- The EVIDENT trial is one of the largest randomised, assessor-blind, controlled trials carried out to date to assess the effectiveness of online depression interventions.

- Strict quality assurance measures were in place to ensure timely data collection and high response rates throughout the course of the trial, leading to high data quality.

- With 1013 participants, the size of the trial allows for robust statistical analyses to explore subgroup differences.

- To ensure robust statistical analyses, some response categories had to be collapsed; while utmost care was applied when choosing the categories, different results may have been found if categories had been collapsed differently.

- Participants were self-selected, which may bias overall effectiveness analyses; however, the moderator analyses reported herein should not have been impacted by recruitment.

Conclusions Our findings suggest that Deprexis is equally beneficial to a wide range of people; that is, participant characteristics were not associated with treatment benefits. Therefore, participant recruitment into web-based psychotherapeutic interventions should be broad, while special attention may be paid to those currently underrepresented in these interventions.

Trial registration number NCT01636752.

\section{INTRODUCTION}

Depressive disorders are a major contributor to the global burden of disease, ${ }^{1}$ with the point prevalence of major depression reported to be as high as $6.9 \%$ in Europe. ${ }^{2}$ Treatment options for depressive disorders generally 
consist of pharmacological and/or psychotherapeutic interventions, with psychotherapeutic interventions traditionally being delivered face-to-face as part of individual or group therapy. In Germany, the main treatment approach includes either cognitive-behavioural therapy (CBT) or psychodynamic psychotherapy, including psychoanalysis. ${ }^{34}$ Both therapeutic approaches have been proven to be effective and efficacious. ${ }^{5}{ }^{6}$ In addition to these more traditional treatment approaches, psychological internet interventions have become increasingly popular, especially over the last two decades, with a large range of evidence-based programmes currently available. These range from internet interventions aimed at health behaviour change to disease prevention as well as treatment. $^{7}$

A prominent example of web-based programme is Deprexis, which was developed in Germany over 10 years ago. ${ }^{8}$ It has been shown to be effective in treating depressive symptoms as demonstrated by a recent meta-analysis that specifically focused on Deprexis, summarising a total of eight studies exploring the effectiveness of this online intervention. ${ }^{9}$ In addition, Deprexis has also been found to be effective in disease areas that are associated with depressive symptoms, such as epilepsy ${ }^{10}$ or multiple sclerosis, ${ }^{11}$ and a large randomised controlled trial (RCT) run in the USA showed that Deprexis is suitable across different cultural contexts. ${ }^{12}$

While there is growing evidence on the effectiveness of web-based interventions for the treatment of depressive symptoms overall, ${ }^{13-16}$ more evidence regarding potential moderating effects of specific participant characteristics is still needed, especially since the current evidence is somewhat ambiguous. One of the largest studies is a recent meta-analysis carried out by Karyotaki et $\mathrm{l}^{17}$ summarising the outcomes of RCTs on self-guided internet-based CBT interventions including data of 3876 participants. No moderating effects of participant-level and study-level variables on treatment effects were found. ${ }^{17}$ Similarly, in an RCT exploring a web-based occupational guided self-help intervention randomising 231 employees into intervention and care as usual (CAU) groups, Geraedts $e t$ $a l^{18}$ did not find any evidence of subgroup differences for sex, age, education, marital status, nationality, working hours and baseline self-reported depressive symptoms. In contrast, Karyotaki et al ${ }^{19}$ published a further metaanalysis based on a sample size of 4889 participants, where older and native-born participants were found to be more likely to respond to the intervention as opposed to their younger counterparts or participants from ethnic minorities. Lundgren $e t a l^{20}$ found that younger age and female sex were associated with more benefits from an internetbased CBT aimed at patients with heart failure and Donker $e t a l^{21}$ also found female sex to be associated with greater treatment benefits. Finally, Høifødt $e t a l^{22}$ reported that married or cohabiting status predicted a more positive treatment response while El Alaoui $e t a l^{33}$ showed employment status (ie, working full time) to be predicting faster recovery and a lower level of post-treatment depressive symptoms. In view of clinical and other potential moderating variables, our group already undertook some subgroup analyses based on EVIDENT data and found that Deprexis seemed most effective in participants who did not take antidepressant medication, especially in the context of medium-term effects, ${ }^{24}$ in those with comorbid social phobia ${ }^{25}$ and in those exhibiting a positive attitude towards internet interventions at baseline. ${ }^{26}$

The aim of the present study was to further investigate whether specific subgroups of participants of the EVIDENT trial benefited more than others from Deprexis, with special focus on a range of sociodemographic variables.

\section{MATERIALS AND METHODS \\ Study design}

To investigate moderating effects in web-based psychotherapy, we used data from the EVIDENT trial, a randomised, assessor-blind, controlled trial that included 1013 participants that was run in Germany between 2012 and 2014. Of these, 509 participants received the online intervention (Deprexis) and CAU, while 504 participants received CAU only (see figure 1 for participants' flow diagram). The sample size calculation was based on the requirements for the main analyses, as reported in the trial protocol; that is, applying an estimated effect size of Cohen's $d=0.23$, power $=0.80$, alpha level $=0.05$ and an anticipated dropout rate of $40 \%$ yielded a sample size requirement of $n=500$ per study arm. ${ }^{27}$ Persons were eligible to join the trial if they were aged between 18 and 65 years and reported mild to moderate depressive symptoms, operationalised as a self-reported score ranging from 5 to 14 inclusive on the Patient Health Questionnaire-9 (PHQ-9).$^{28}$ Additional email support was offered to the intervention group subjects with a PHQ-9 score of $\geq 10$ points at study inclusion. Outcomes were assessed at baseline, 12 weeks (postintervention), 6 months (first follow-up) and 12 months (second follow-up). An independent investigator managed the group allocation schedule by applying a computerised random number generator using variable block sizes and further performing stratification by depression severity to ensure equal allocation of disease severity to each study arm. All other investigators were blinded to allocation sequence. ${ }^{27}$

When writing this report, we used the Consolidated Standards of Reporting Trials (CONSORT) ${ }^{29}$ and the CONSORT-EHEALTH ${ }^{30}$ checklists. To avoid duplication, some details that were not critical for the present article (eg, further details on trial design, participant recruitment, study execution) are reported in the EVIDENT trial protocol ${ }^{27}$ and the core paper reporting on the main results of the EVIDENT study. ${ }^{31}$

\section{Intervention}

Deprexis is an integrative, web-based, individually tailored programme for the treatment of depressive disorders. Its curriculum is mainly based on CBT methods and covers 


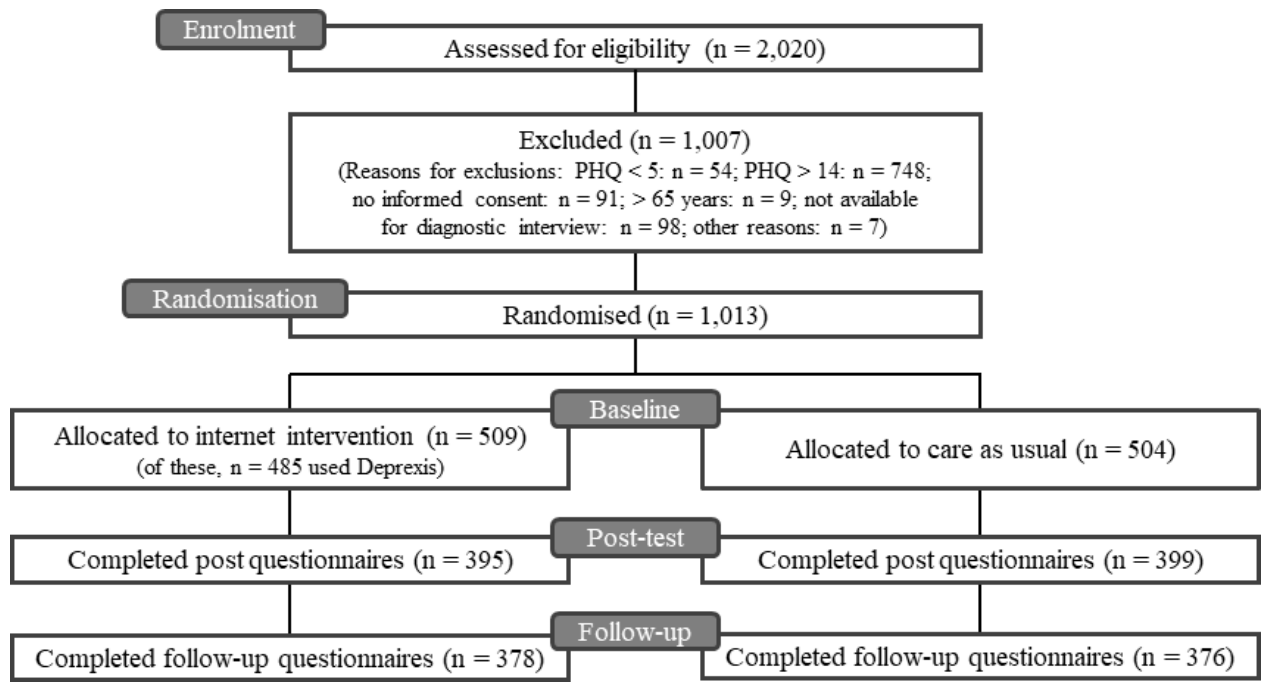

Figure 1 Participant flow diagram. PHQ-9, Patient Health Questionnaire-9.

a range of therapeutic approaches, including cognitive restructuring, behavioural activation and mindfulness/ acceptance exercises.

\section{Patient and public involvement}

As part of the EVIDENT study, we did not involve patients or members of the public in the design, conduct, reporting or dissemination of the research.

\section{Participant recruitment}

Participant recruitment was broad across various regions in Germany. It included recruitment in inpatient and outpatient clinics (predominantly general practice and psychological clinics) through clinicians, as well as distribution of marketing materials (flyers, posters), advertisements in online depression forums, direct marketing to insurees via health insurance companies and local media (newspapers, radio). Informed consent from each participant was obtained online prior to baseline assessment.

\section{Data collection}

All self-reported data were collected electronically. Strict quality assurance measures were in place to ensure timely data collection and high response rates throughout the course of the trial. These included participants receiving email reminders for the postintervention and the two follow-up assessment time points. In case of non-response, study participants were followed up twice at respective time points. Study materials for each study centre included time sheets with deadlines to ensure that data were collected at or closely around each predefined data collection time point following the online intervention, that is, postintervention (12 weeks after baseline) as well as 6-month and 12-month follow-up.

\section{Outcome variables}

The primary endpoint of the EVIDENT trial was change in self-rated depression severity between baseline and postintervention (12 weeks after baseline assessment) as measured by the PHQ-9. The PHQ-9 consists of nine items measuring depression severity. For each item, respondents are asked to rate their perceived symptom burden (frequency) during the past 2 weeks. Scoring is between 0 (not at all) and 3 (nearly every day), with the PHQ-9 total score ranging between 0 and 27 points. ${ }^{28}$ Change in depression severity was calculated such that positive values indicate improvement (decrease in PHQ-9 scores) and negative values indicate deterioration (increase in PHQ-9 scores) between baseline and postintervention.

\section{Sociodemographic and self-reported clinical variables}

Sociodemographic variables assessed as part of the EVIDENT trial included sex (female/male), age (in years), educational attainment (lower secondary school, middle secondary school, higher secondary school qualifying for a university of applied sciences, higher secondary school qualifying for university (German: 'Abitur'), other), employment status (full-time employed, part-time employed, not working (including students, unemployed, retirees), other) and relationship status (married/registered partnership and living together, married/registered partnership but not living together, in stable relationship, single, divorced, widower). In addition, we collected self-reported 'lifetime number of depressive episodes', including the current depressive episode.

\section{Statistical analyses}

Mean baseline and post-test assessment scores are reported for the intervention and control group subjects separately. In addition, between-group differences were calculated using Cohen's $d{ }^{32}$ including the $95 \%$ CI of the effect size estimate. Cohen's $d$ was determined by calculating the difference score between the mean scores of the control and intervention groups and then dividing the difference by the pooled standard deviation (SD) of the two groups. Interpretation of $d$ follows Cohen's suggestion of $d=0.2$ considered a small, $d=0.5$ considered a medium and $d=0.8$ considered a large effect size. ${ }^{32}$ 
Potential moderating effects were investigated using linear regression models based on intention-to-treat (ITT) analyses. The models included change in PHQ-9 from baseline to postintervention assessment as the outcome, group allocation as a predictor and baseline PHQ-9 score as a covariate. As moderator variables, we chose the same sociodemographic variables as selected by Karyotaki et $a l^{17}$ in their meta-analysis, including sex, age, educational attainment, employment status and relationship status. In addition, we included the variable 'lifetime frequency of depressive episodes'. Given the small number of observations in some response categories of the proposed moderator variables, a number of moderators were recoded by aggregating response options into overarching but interpretable categories. That is, educational attainment was dichotomised into 'Abitur' vs 'else', as upper secondary school qualification, that is, reaching the formal university entrance qualification, is one of the strongest predictors of social class (including risk of poverty and social inequality) and health (eg, health status and healthdirected behaviour) in Germany. ${ }^{33}$ Employment was dichotomised into 'full-time/part-time employed' versus 'else'. Relationship status was recoded into 'married/ in a stable relationship', 'not living with a partner' and 'single'. Finally, 'lifetime frequency of depressive episodes' was recoded as ' 1 episode', ' $2-5$ episodes', '6-10 episodes', '11-20 episodes' and '>20 episodes'.

The strength of associations between change in PHQ-9 and each of the variables in the model was assessed with regression coefficients $\beta$. The higher and positive values of $\beta$ denote a more favourable effect (greater decrease in the severity of depression). Before carrying out the statistical analyses, assumptions underlying the use of a linear regression, including linearity, homoscedasticity, noncollinearity and normality of residuals, were checked. While no violations of linearity, normality and homoscedasticity assumptions were detected, provision of email support, which was initially considered to be included in the model, was a strong contributor to collinearity due to overlap with group allocation and baseline PHQ-9 scores. Therefore, this variable was excluded from further analyses. In all analyses, a statistical significance level alpha of 0.05 was used (two-tailed tests).

Moderating effects were assessed with tests of multiplicative interactions between group allocation and moderator variables. To reduce potential collinearity problems, the continuous variable 'age' was mean-centred before computing the interaction terms.

Model building to test moderating effects proceeded in three steps. The initial model contained the intervention group, baseline PHQ-9 and all moderator variables as fixed factors. In the next steps, interactions between group allocation and a moderator were added to the model, with separate models tested for each moderator. Once all moderating effects were tested individually, all significant moderating effects were then entered into the last and final model simultaneously to assess their relative contribution to the outcome.

\section{Handling of missing data}

Multiple imputation by chained equations was used to handle missing data, with 25 imputed data sets created for the analyses. Relative efficiency estimates for the 25 imputed data sets were at least $99 \%$ for all model coefficients, which is considered sufficient. All moderator variables (sex, age, educational attainment, employment status, relationship status and lifetime frequency of episodes), group allocation, provision of email support, PHQ-9 baseline scores and multiplicative interactions between group allocation and moderator variables were included in the imputation model. In addition, we included the baseline Medical Outcomes Study (MOS) 36-item Short-Form Health Survey mental and physical component summary scores ${ }^{34}$ to improve accuracy of imputed values.

\section{RESULTS}

\section{Study population}

Of the 1013 participants of the EVIDENT study, 31\% were male; the average age was 43 years. About $60 \%$ were married/living with a partner and about half of the participants had obtained a university entrance qualification ('Abitur'). About two-thirds were either working full time or part time, and the most frequently reported lifetime frequency of depressive episodes was between two and five episodes (see table 1). Further details on the clinical characteristics of the study sample are described elsewhere. ${ }^{31}$

As reported in detail in the core paper, ${ }^{31}$ the noncompletion rate at post-test assessment was $21.6 \%$ across groups. Using logistic regression analyses, it was shown that patient dropout was not associated with any of the following variables: group allocation, sex, age, relationship status, educational attainment, baseline PHQ-9 score, baseline diagnosis of depression (clinician-reported) or self-reported panic disorder. ${ }^{31}$

\section{Mean PHQ-9 scores at baseline and postintervention assessment}

As already reported in detail in the core paper of the EVIDENT trial, ${ }^{31}$ the mean baseline score was around 10 points on the PHQ-9 (table 2), which is the cut-off between mild and moderate depressive symptoms. ${ }^{28}$ At post-test, that is, 12 weeks after baseline, group differences between intervention and control group subjects were observed, with Cohen's $d$ indicating small-to-mediumsized between-group effects.

As we were mostly interested in subgroup differences, the between-group effects for the subgroups were explored more closely. As shown in table 2, there were no apparent differences in the magnitude of Cohen's $d$ within each subgroup, with the largest differences observed in the subgroup 'relationship status', with those not living with a partner showing medium size effects, while the other two groups (ie, married/living with a partner; single) showing small size effects. 
Table 1 Sociodemographic characteristics of the participants of the EVIDENT trial: comparison of intervention group and control group

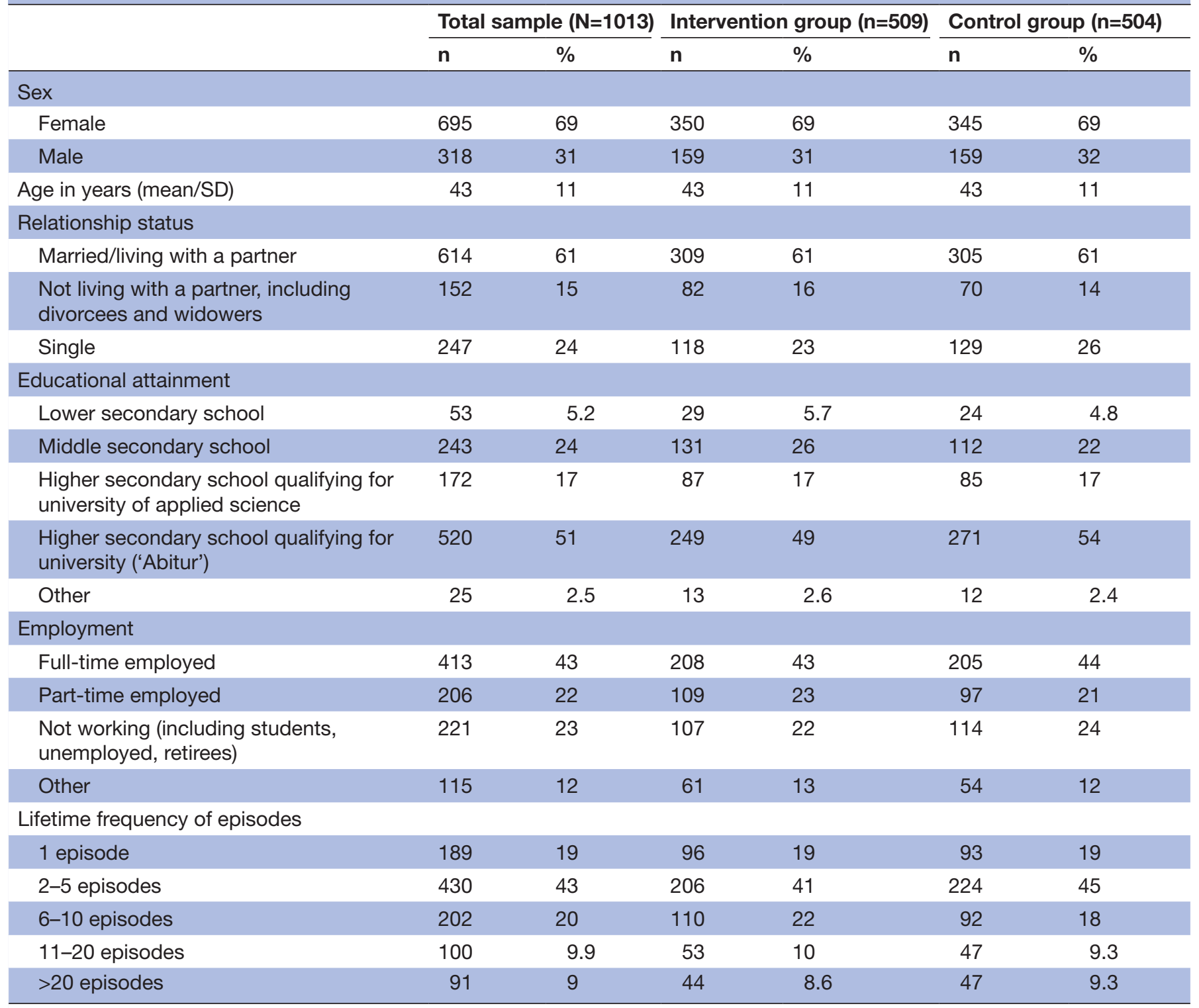

$\mathrm{SD}$, standard deviation.

\section{Main analyses}

The results of the linear regression models assessing the moderating effects of sociodemographic variables and lifetime frequency of depressive episodes on the outcome of the intervention are summarised in table 3. Overall, individuals in the intervention group experienced a significantly larger decrease in depression severity than control group subjects $(\beta=1.75,95 \%$ CI 1.19 to $2.31, \mathrm{p}<0.001)$, after adjusting for baseline PHQ-9 and age, sex, education, employment, marital status and frequency of depressive episodes. A greater reduction in depression severity was also associated with higher (worse) baseline PHQ-9 scores $(\beta=0.55,95 \%$ CI 0.43 to $0.66, p<0.001)$, while individuals who reported $>20$ lifetime depressive episodes showed a significantly smaller decrease in depression severity than those who reported one depressive episode $(\beta=-1.84,95 \% \mathrm{CI}-3.05$ to $-0.63, p=0.003)$. For the group of participants with between 6 and 10 episodes, there was a trend towards fewer benefits compared with those with one depressive episode, with the upper bound of the $95 \%$ CI close to zero $(\beta=-0.86,95 \%$ CI -1.75 to 0.02 , $\mathrm{p}=0.06$ ). As shown in table 3 , step 2 , however, none of the examined moderator tests reached statistical significance, indicating that the magnitude of the intervention effect was not influenced by age and was homogenous across groups defined by sex, educational attainment, employment status, marital status as well as lifetime frequency of depressive episodes.

\section{DISCUSSION}

In the present study, we set out to investigate the potential moderating effects of a range of sociodemographic 
Table 2 PHQ-9 scores at baseline and at 12 weeks post assessment in the EVIDENT trial: intervention group $(n=509)$ versus care as usual control group $(n=504)$

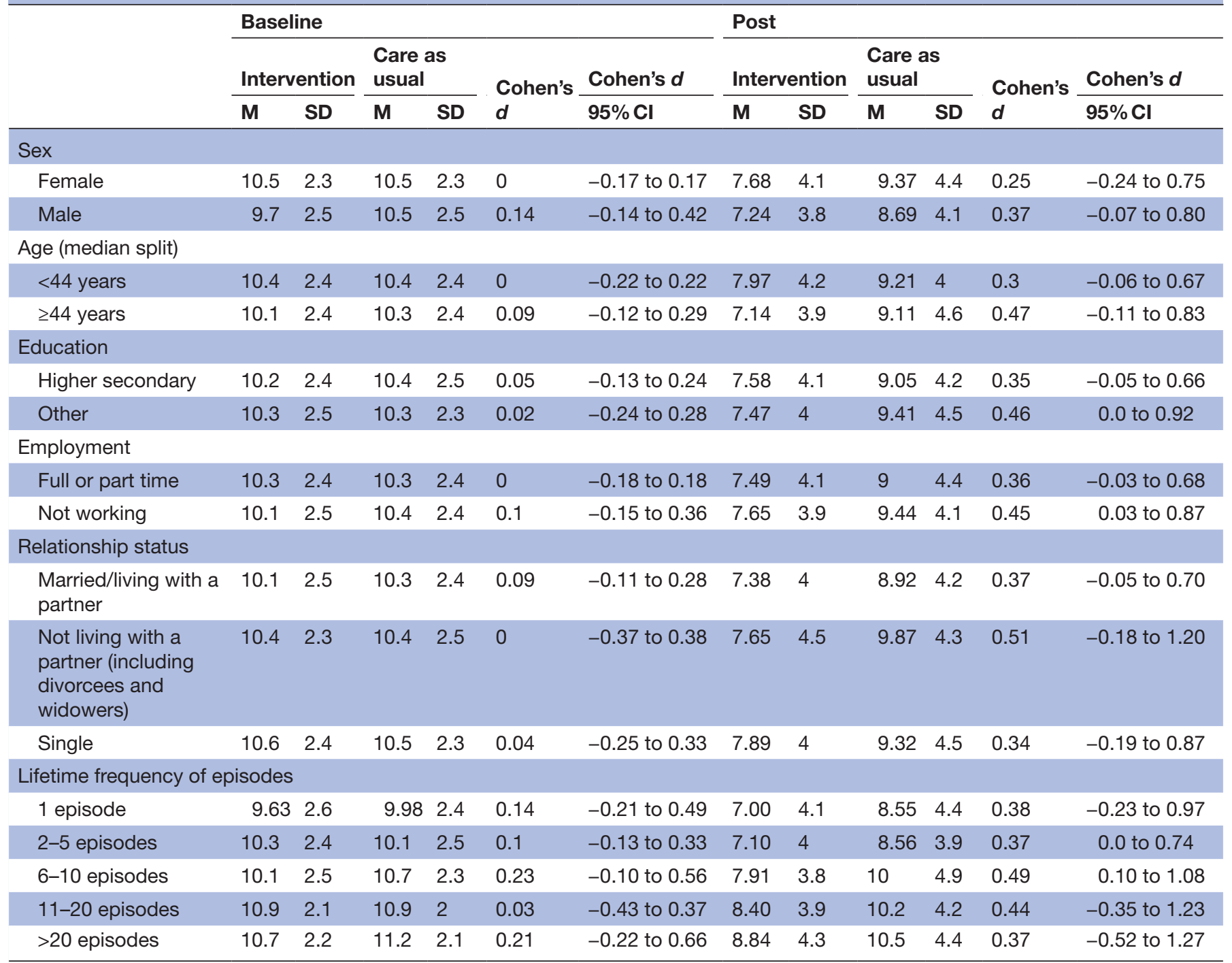

Cl, confidence interval; M, mean; PHQ-9, Patient Health Questionnaire-9; SD, standard deviation.

variables as well as variable 'lifetime frequency of depressive episodes' on the outcomes of participants of Deprexis, an online CBT-based intervention for the treatment of depressive symptoms. While baseline scores were predictive of symptom change, with higher baseline scores (ie, higher degrees of depressive symptom burden) associated with greater reduction of depressive symptoms, none of the included sociodemographic and clinical variables was found to be moderating depression outcomes based on the ITT population. Our findings therefore suggest that an online depression intervention is equally beneficial to a large range of participants. This is a reassuring finding and implies that the intervention is suitable for many people with mild to moderate depressive symptoms regardless of the participant characteristics we examined here.

To put our results in context, the current evidence regarding potential moderating effects of participant characteristics in web-based depression interventions is inconclusive. By and large, we confirmed the findings of Karyotaki $e t a l^{17}$ and Geraedts $e t a l,{ }^{18}$ who also did not find any moderating effects of participant-level and studylevel variables on treatment outcomes. That is, regardless of sex, age, educational attainment, employment status, relationship status and self-reported lifetime frequency of depressive episodes, participants seemed to have received comparable benefits from using the web-based depression intervention Deprexis.

\section{Clinical implications}

The finding that many participants benefited from engaging in an online depression course suggests that it seems sensible to recommend broad recruitment strategies to attract a wide range of persons with mild to moderate depressive symptoms to web-based depression interventions. This finding is particularly important in the context of those that may currently be under-represented in online courses. For example, we found that persons with 
Table 3 Results of the linear regression models based on intention-to-treat analyses

\begin{tabular}{|c|c|c|c|}
\hline & $\beta$ & $95 \% \mathrm{Cl}$ & $P$ value \\
\hline \multicolumn{4}{|l|}{ Step 1: main effects } \\
\hline \multicolumn{4}{|l|}{ Intervention group } \\
\hline Control & \multicolumn{3}{|c|}{ Reference } \\
\hline Intervention & 1.75 & 1.19 to 2.31 & $<0.001$ \\
\hline $\begin{array}{l}\text { PHQ-9 total score at } \\
\text { baseline }\end{array}$ & 0.55 & 0.43 to 0.66 & $<0.001$ \\
\hline Age in years & 0.01 & -0.02 to 0.04 & 0.44 \\
\hline \multicolumn{4}{|l|}{ Sex } \\
\hline Male & \multicolumn{3}{|c|}{ Reference } \\
\hline Female & -0.38 & -1.02 to 0.25 & 0.24 \\
\hline \multicolumn{4}{|l|}{ Education } \\
\hline $\begin{array}{l}\text { High school } \\
\text { ('Abitur', ie, reaching } \\
\text { university entrance } \\
\text { qualification) }\end{array}$ & \multicolumn{3}{|c|}{ Reference } \\
\hline Other & 0.1 & -0.51 to 0.71 & 0.75 \\
\hline Employment & & & \\
\hline
\end{tabular}

Full-time or part-time Reference employed

\begin{tabular}{|c|c|c|c|}
\hline Not working & 0.12 & -0.47 to 0.71 & 0.69 \\
\hline \multicolumn{4}{|l|}{ Marital group } \\
\hline $\begin{array}{l}\text { Married/in a stable } \\
\text { relationship }\end{array}$ & \multicolumn{3}{|c|}{ Reference } \\
\hline $\begin{array}{l}\text { Not living with a } \\
\text { partner, including } \\
\text { divorcees to } \\
\text { widowers }\end{array}$ & -0.07 & -0.96 to 0.82 & 0.87 \\
\hline Single & -0.32 & -1.05 to 0.4 & 0.38 \\
\hline \multicolumn{4}{|l|}{ Frequency of episodes } \\
\hline 1 episode & \multicolumn{3}{|c|}{ Reference } \\
\hline $2-5$ episodes & -0.12 & -0.89 to 0.64 & 0.75 \\
\hline $6-10$ episodes & -0.86 & -1.75 to 0.02 & 0.06 \\
\hline 11-20 episodes & -0.91 & -1.96 to 0.14 & 0.09 \\
\hline$>20$ episodes & -1.84 & -3.05 to -0.63 & 0.003 \\
\hline \multicolumn{4}{|c|}{ Step 2: moderator effects } \\
\hline Intervention by age & 0.01 & -0.04 to 0.06 & 0.56 \\
\hline Intervention by sex & -0.05 & -1.27 to 1.17 & 0.94 \\
\hline $\begin{array}{l}\text { Intervention by } \\
\text { education }\end{array}$ & 0.89 & -0.21 to 1.99 & 0.11 \\
\hline $\begin{array}{l}\text { Intervention by } \\
\text { employment }\end{array}$ & 0.16 & -0.95 to 1.27 & 0.77 \\
\hline $\begin{array}{l}\text { Intervention by marital } \\
\text { group (overall effect) }\end{array}$ & & & 0.32 \\
\hline $\begin{array}{l}\text { Intervention by } \\
\text { frequency of episodes } \\
\text { (overall effect) }\end{array}$ & & & 0.95 \\
\hline
\end{tabular}

$P$ values marked in bold are significant at the $p<0.05$ level. $\mathrm{Cl}$, confidence interval; PHQ-9, Patient Health Questionnaire-9. lower educational attainment were under-represented in the EVIDENT study, ${ }^{35}$ a phenomenon that has also been observed in other psychotherapy studies. ${ }^{36}$ Similarly, men were under-represented in our study, with a participation ratio of women versus men of 2:1. As those with lower educational attainment as well as men seem to have benefited just as much from Deprexis compared with those more likely to participate (eg, more highly educated individuals, women), it seems further reasonable to recommend that recruitment strategies could particularly target those who seem to be less frequent attendees. For example, recruitment outside of RCTs, such as medical practices, has been shown to be a very effective way to reach populations with lower educational attainment compared with the present sample. ${ }^{37}$ However, in this context it needs to be considered that our results are based on a sample that consisted of self-selected individuals; that is, as is the case with many self-management type interventions, results may be biased towards participants who self-selected into these courses. ${ }^{38}$ Therefore, it is likely that participants were highly motivated at the start of the intervention and were ready to change. ${ }^{39}$ Self-selection bias, however, is most problematic in the context of observational studies. In an RCT such as the EVIDENT trial, both motivation and readiness to change can be assumed to be comparable between intervention and control group subjects; hence, observed group differences at the end of the trial may be mostly due to the intervention rather than other competing reasons. Also, research suggests that selfselection may not be a large issue in depression internet interventions. ${ }^{40}$ However, it remains that recruitment of those under-represented may prove difficult if the reason for non-participation is lack of motivation.

\section{Limitations}

Our study has some limitations. First, the statistical power for subgroup analyses is lower compared with main effect analyses, particularly if subgroups are not identical in size, as in the present study. ${ }^{41}$ Therefore, the absence of a statistically significant moderating effect does not necessarily mean that it applies to all subgroups. ${ }^{42}$ However, to date the EVIDENT study is one of the largest studies carried out in this area; that is, the chosen subgroup categories enabled us to carry out these types of analyses, giving us confidence that the lack of moderating effects is not a false-negative finding. Second, despite careful selection of potential moderating variables, this post-hoc analysis was limited to those variables that were assessed as part of the EVIDENT trial, a trial that was designed to answer a different research question from ours. ${ }^{27} 31$ Therefore, it cannot be ruled out that other variables that were not assessed indeed moderated treatment outcomes, for example, ethnicity, language ability or health literacy. However, we feel the selected sociodemographic variables cover a reasonable range of participant characteristics and are in line with the sociodemographic variables examined in a recent meta-analysis. ${ }^{17}$ Third, the response categories of some of the variables had to be collapsed to 
ensure sufficient numbers of observations in subgroups of moderator variables. If response categories had been collapsed in different ways, it cannot be ruled out that this would have led to different results. However, we carefully selected the chosen categories and are confident that these were sensible to answer the present research question.

\section{CONCLUSIONS}

In conclusion, to our knowledge the EVIDENT trial is one of the largest RCTs to date exploring the effects of an online depression intervention. Results suggest that the web-based course Deprexis significantly improved depression outcomes in the treatment group and these were not moderated by sex, age, educational attainment, employment status, relationship status or lifetime frequency of depressive episodes. Therefore, recruitment of participants to online psychotherapeutic interventions should be broad, while special attention may be paid to those currently under-represented in web-based depression courses as well as those who may not seek any type of psychotherapeutic treatment whether it is delivered online or face-to-face.

\section{Author affiliations}

${ }^{1}$ Medical Department, Division of Psychosomatic Medicine, Charité -

Universitätsmedizin Berlin, corporate member of Freie Universität Berlin, HumboldtUniversität zu Berlin, and Berlin Institute of Health, Berlin, Germany

${ }^{2}$ Research Methodology Division, Department of Epidemiology and Preventive

Medicine, School of Public Health and Preventive Medicine, Monash University, Melbourne, Victoria, Australia

${ }^{3}$ Department of Clinical Psychology and Psychotherapy, University of Bern, Bern, Switzerland

${ }^{4}$ Research Department, GAIA AG, Hamburg, Germany

${ }^{5}$ Department of Psychology, City University London, London, UK

${ }^{6}$ Department of Psychiatry and Psychotherapy, University Medical Center HamburgEppendorf, Hamburg, Germany

${ }^{7}$ Department of Psychiatry and Psychotherapy, Lübeck University, Lübeck, Germany

Acknowledgements The authors wish to thank GAIA AG (Hamburg, Germany) for technical support and making the internet intervention (Deprexis) available at no cost for participants in the trial. The full EVIDENT study team consists of Sandra Nolte, Matthias Rose (local principal investigator), Anna Paulitschek, Leonie Gmöhling and Leonie Schickedanz (Berlin, Germany); Thomas Berger (Bern, Switzerland); Viola Gräfe and Wolfgang Greiner (local principal investigator) (Bielefeld, Germany); Mirja Behrens, Cecile Hoermann, Anna J Katharina Jahns, Thies Lüdtke, Björn Meyer, Steffen Moritz (local principal investigator), Johanna Schröder, Amit Gulati and Eik Vettorazzi (Hamburg, Germany); Carla Gamon, Fritz Hohagen, Martin Kolbe, Philipp Klein (local principal investigator), Antje Roniger and Christina Späth-Nellissen (Lübeck, Germany); Alice Arndt, Liv Glindemann, Wolfgang Lutz (local principal investigator), David Rosenbaum and Kathinka Wolter (Trier, Germany); Flora Bach, Elisabeth Beck, Kristina Fuhr, Martin Hautzinger (local principal investigator), Katharina Krisch and Melanie Wahl (Tübingen, Germany).

Contributors SN developed the analysis plan, interpreted the results of the analyses and drafted the overall manuscript in cooperation with JPK. LB developed the analysis plan, carried out the statistical analyses and data interpretation, and drafted the analysis sections of the manuscript. TB, BM, SM and JPK designed the EVIDENT study and obtained funding. The EVIDENT study steering committee (TB, $\mathrm{BM}, \mathrm{SM}, \mathrm{JS}, \mathrm{CS}-\mathrm{N}$ and JPK) coordinated patient recruitment and assessments. MR was the local principal investigator and critically reviewed the manuscript. All authors commented on the manuscript and approved the final version.
Funding This work was supported by the German Federal Ministry of Health (grant number: IIA5-2512 FSB 052). The funding body had no role in the design of the study, data collection, analysis or interpretation of the data.

Competing interests JPK has received payments for presentations, workshops and books on psychotherapy for chronic depression and on psychiatric emergencies. BM is employed as research director at GAIA AG, the company that developed, owns and operates the internet intervention investigated in this trial. All other authors report no conflicts of interests.

Patient consent for publication Not required.

Ethics approval The trial was conducted in compliance with the Declaration of Helsinki. Ethical approval was obtained from the Ethics Committee of the German Psychological Association (SM 04_2012).

Provenance and peer review Not commissioned; externally peer reviewed.

Data availability statement Data are available upon reasonable request from the last author of this manuscript (Philipp.Klein@uksh.de).

Open access This is an open access article distributed in accordance with the Creative Commons Attribution Non Commercial (CC BY-NC 4.0) license, which permits others to distribute, remix, adapt, build upon this work non-commercially, and license their derivative works on different terms, provided the original work is properly cited, appropriate credit is given, any changes made indicated, and the use is non-commercial. See: http://creativecommons.org/licenses/by-nc/4.0/.

\section{ORCID iDs}

Sandra Nolte http://orcid.org/0000-0001-6185-9423

Thomas Berger http://orcid.org/0000-0002-2432-7791

Jan Philipp Klein http://orcid.org/0000-0001-9882-2261

\section{REFERENCES}

1 Vos T, Flaxman AD, Naghavi M, et al. Years lived with disability (YLDs) for 1160 sequelae of 289 diseases and injuries 1990-2010: a systematic analysis for the global burden of disease study 2010 . Lancet 2012;380:2163-96.

2 Wittchen HU, Jacobi F, Rehm J, et al. The size and burden of mental disorders and other disorders of the brain in Europe 2010. Eur Neuropsychopharmacol 2011;21:655-79.

3 Härter M, Klesse C, Bermejo I. Unipolar depression. Dtsch Arztebl International 2010;107:700-8.

4 Zipfel S, Herzog W, Kruse J, et al. Psychosomatic medicine in Germany: more timely than ever. Psychother Psychosom 2016;85:262-9.

5 Abbass AA, Hancock JT, Henderson J. Short-term psychodynamic psychotherapies for common mental disorders. Cochrane Database Syst Rev 2006;4:50.

6 Lambert MJ. The efficacy and effectiveness of psychotherapy. New York: John Wiley \& Sons, 2013: 169-218.

7 Rogers MA, Lemmen K, Kramer R, et al. Internet-delivered health interventions that work: systematic review of meta-analyses and evaluation of website availability. J Med Internet Res 2017;19:e90.

8 Meyer B, Berger T, Caspar F, et al. Effectiveness of a novel integrative online treatment for depression (Deprexis): randomized controlled trial. J Med Internet Res 2009;11:e15.

9 Twomey C, O'Reilly G, Meyer B. Effectiveness of an individuallytailored computerised CBT programme (Deprexis) for depression: a meta-analysis. Psychiatry Res 2017;256:371-7.

10 Schröder J, Brückner K, Fischer A, et al. Efficacy of a psychological online intervention for depression in people with epilepsy: a randomized controlled trial. Epilepsia 2014;55:2069-76.

11 Fischer A, Schröder J, Vettorazzi E, et al. An online programme to reduce depression in patients with multiple sclerosis: a randomised controlled trial. Lancet Psychiatry 2015;2:217-23.

12 Beevers CG, Pearson R, Hoffman JS, et al. Effectiveness of an internet intervention (Deprexis) for depression in a United States adult sample: a parallel-group pragmatic randomized controlled trial. J Consult Clin Psychol 2017;85:367-80.

13 Saddichha S, Al-Desouki M, Lamia A, et al. Online interventions for depression and anxiety - a systematic review. Health Psychol Behav Med 2014;2:841-81.

14 Zhou T, Li X, Pei Y, et al. Internet-based cognitive behavioural therapy for subthreshold depression: a systematic review and metaanalysis. BMC Psychiatry 2016;16:356. 
15 Josephine K, Josefine L, Philipp D, et al. Internet- and mobilebased depression interventions for people with diagnosed depression: a systematic review and meta-analysis. J Affect Disord 2017;223:28-40.

16 Păsărelu CR, Andersson G, Bergman Nordgren L, et al. Internetdelivered transdiagnostic and tailored cognitive behavioral therapy for anxiety and depression: a systematic review and meta-analysis of randomized controlled trials. Cogn Behav Ther 2017;46:1-28.

17 Karyotaki E, Riper H, Twisk J, et al. Efficacy of self-guided Internetbased cognitive behavioral therapy in the treatment of depressive symptoms: a meta-analysis of individual participant data. JAMA Psychiatry 2017;74:351-9.

18 Geraedts AS, Kleiboer AM, Twisk J, et al. Long-Term results of a web-based guided self-help intervention for employees with depressive symptoms: randomized controlled trial. J Med Internet Res 2014;16:e168.

19 Karyotaki E, Ebert DD, Donkin L, et al. Do guided Internet-based interventions result in clinically relevant changes for patients with depression? An individual participant data meta-analysis. Clin Psychol Rev 2018;63:80-92.

20 Lundgren JG, Dahlström Örjan, Andersson G, et al. The effect of guided web-based cognitive behavioral therapy on patients with depressive symptoms and heart failure: a pilot randomized controlled trial. J Med Internet Res 2016;18:e194.

21 Donker T, Batterham PJ, Warmerdam L, et al. Predictors and moderators of response to internet-delivered interpersonal psychotherapy and cognitive behavior therapy for depression. $J$ Affect Disord 2013;151:343-51.

22 Høifødt RS, Mittner M, Lillevoll K, et al. Predictors of response to web-based cognitive behavioral therapy with high-intensity face-toface therapist guidance for depression: a Bayesian analysis. J Med Internet Res 2015;17:e197.

23 El Alaoui S, Ljótsson B, Hedman E, et al. Predicting outcome in Internet-based cognitive behaviour therapy for major depression: a large cohort study of adult patients in routine psychiatric care. PLoS One 2016;11:e0161191.

24 Klein JP, Späth C, Schröder J, et al. Time to remission from mild to moderate depressive symptoms: one year results from the EVIDENTstudy, an RCT of an internet intervention for depression. Behav Res Ther 2017;97:154-62.

25 Probst T, Berger T, Meyer B, et al. Social phobia moderates the outcome in the EVIDENT study: a randomized controlled trial on an Internet-based psychological intervention for mild to moderate depressive symptoms. J Consult Clin Psychol 2020;88:82-9.

26 Schröder J, Berger T, Meyer B, et al. Impact and change of attitudes toward Internet interventions within a randomized controlled trial on individuals with depression symptoms. Depress Anxiety 2018;35:421-30.

27 Klein JP, Berger T, Schröder J, et al. The EVIDENT-trial: protocol and rationale of a multicenter randomized controlled trial testing the effectiveness of an online-based psychological intervention. BMC Psychiatry 2013;13:239.

28 Kroenke K, Spitzer RL, Williams JB. The PHQ-9: validity of a brief depression severity measure. J Gen Intern Med 2001;16:606-13.

29 Schulz KF, Altman DG, Moher D, et al. CONSORT 2010 statement: updated guidelines for reporting parallel group randomised trials. BMJ 2010;340:c332.

30 Eysenbach G, CONSORT-EHEALTH Group. CONSORT-EHEALTH: improving and standardizing evaluation reports of web-based and mobile health interventions. J Med Internet Res 2011;13:e126.

31 Klein JP, Berger T, Schröder J, et al. Effects of a psychological Internet intervention in the treatment of mild to moderate depressive symptoms: results of the evident study, a randomized controlled trial. Psychother Psychosom 2016;85:218-28.

32 Cohen J. Statistical power analysis for the behavioural sciences. 2nd edn. Hillsdale, NJ: Lawrence Erlbaum Associates, 1988.

33 Lampert T, Saß A, Häfelinger M. Armut, soziale Ungleichheit und Gesundheit. expertise des Robert Koch-Instituts zum 2 Armuts- und Reichtumsbericht Der Bundesregierung, 2005.

34 Ware JE, Sherbourne CD. The mos 36 -item short-form health survey (SF-36). I. conceptual framework and item selection. Med Care 1992;30:473-81.

35 Späth C, Hapke U, Maske U, et al. Characteristics of participants in a randomized trial of an Internet intervention for depression (evident) in comparison to a national sample (DEGS1). Internet Interv 2017:9:46-50.

36 Schramm E, Kriston L, Zobel I, et al. Effect of disorder-specific vs nonspecific psychotherapy for chronic depression: a randomized clinical trial. JAMA Psychiatry 2017;74:233-42.

37 Klein JP, Barthel B, Berger T, et al. Feasibility, effectiveness and safety of the self-management intervention deprexis in routine medical care: results of an uncontrolled observational study. Internet Interv 2020;22:100341.

38 Nolte S, Elsworth GR, Sinclair AJ, et al. The extent and breadth of benefits from participating in chronic disease self-management courses: a national patient-reported outcomes survey. Patient Educ Couns 2007;65:351-60.

39 Prochaska JO, Redding CA, Evers KE. The transtheoretical model and stages of change. In: Glanz K, Lewis FM, Rimer BK, eds. Health behavior and health education: theory, research, and practice. 4th edn. San Francisco: John Wiley \& Sons, Inc, 2008: 97-122.

40 Donkin L, Hickie IB, Christensen H, et al. Sampling bias in an internet treatment trial for depression. Transl Psychiatry 2012;2:e174.

41 Brookes ST, Whitely E, Egger M, et al. Subgroup analyses in randomized trials: risks of subgroup-specific analyses; power and sample size for the interaction test. J Clin Epidemiol 2004;57:229-36.

42 Wang R, Lagakos SW, Ware JH, et al. Statistics in medicine-reporting of subgroup analyses in clinical trials. $N$ Engl J Med 2007;357:2189-94. 